\title{
Capsaicin-capped silver nanoparticles: its kinetics, characterization and biocompatibility assay
}

\author{
Nagoth Joseph Amruthraj • \\ John Poonga Preetam Raj • \\ Antoine Lebel
}

Received: 22 March 2014/ Accepted: 16 June 2014/ Published online: 6 July 2014

(C) The Author(s) 2014. This article is published with open access at Springerlink.com

\begin{abstract}
Capsaicin was used as a bio-reductant for the reduction of silver nitrate to form silver nanoparticles. The formation of the silver nanoparticles was initially confirmed by color change and Tyndall effect of light scattering. It was characterized with UV-visible spectroscopy, FTIR and TEM. Hemagglutination (H) test and H-inhibition assay were performed in the presence of AgNPscapsaicin conjugates. The silver colloid solution after complete reduction turned into pale gray color. The characteristic surface plasmon resonance of silver nanoparticles (SNPs) was observed at $450 \mathrm{~nm}$. Time taken for complete bio-reduction of silver nitrate and capping was found to be 16 hours. The amount of capsaicin required to reduce $20 \mathrm{ml}$ of $1 \mathrm{mM}$ silver nitrate solution was found to be $40 \mu \mathrm{g}$ approximately. The FTIR results confirmed the capping of capsaicin on the silver metal. The particle size was within the range of $20-30 \mathrm{~nm}$. The hemagglutination and $\mathrm{H}$-inhibition test was negative for all the blood groups. The capsaicin-capped silver nanoparticles were compatible with blood cells in hemagglutination test implying biocompatibility as future therapeutic drug.
\end{abstract}

Keywords Capsaicin - Silver nanoparticles (SNPs) · $\mathrm{AgNO}_{3} \cdot$ Bio-reductant $\cdot$ Biocompatibility .

Hemagglutination

N. J. Amruthraj · J. P. Preetam Raj $(\bowtie) \cdot$ A. Lebel

Department of Plant Biology and Biotechnology, Loyola

College, Chennai 600 034, Tamil Nadu, India

e-mail: preetamraj.jp@gmail.com

\section{Introduction}

Nanotechnology has a massive impact on therapeutic drugs. It significantly improves the performance of drugs in terms of efficacy and safety. Wide range of nano materials can be designed with surface modification to tag variety of chemical, molecular and biological entities (Kumari et al. 2012; Baimark 2012). Capping agents that bind to the nanoparticles surface will improve stability, water solubility, biocompatibility, specificity and prevent aggregation (Sing et al. 2009).

Silver has been used for medicinal purpose in the field of health care from ancient period (Priyaragini et al. 2013). The reduction of silver nitrate to silver nanoparticles was described by (Jin et al. 2001). "Green synthesis" of nanoparticles is a beneficial method that makes use of non-toxic reagents. Silver nanoparticles have tremendous scope, because of their important applications in antimicrobial, catalysis and surface-enhanced Raman scattering ( $\mathrm{Li}$ et al. 2006; Chen et al. 2005; Setua et al. 2007). Silver-based drugs have proved as antimicrobial agents. The rapid breakdown of silver nanoparticles releases ionic silver that inactivates vital bacterial enzymes by interacting with essential thiol groups. Silver ions can inhibit bacterial DNA replication, damage bacterial cytoplasmic membranes, deplete the levels of intracellular adenosine triphosphate (ATP) and finally cause cell death (Feng et al. 2000).

Plant-derived drugs have served as the base for an enormous fraction of the current pharmacopoeia (Kingston and Newman 2002). Plant-based therapeutic molecules as drugs have limitations due to reduced solubility, permeability and bioavailability (Kumari et al. 2012). In spite of the challenges, the following natural products like artemisinin, curcumin, triptolide and capsaicin have been 


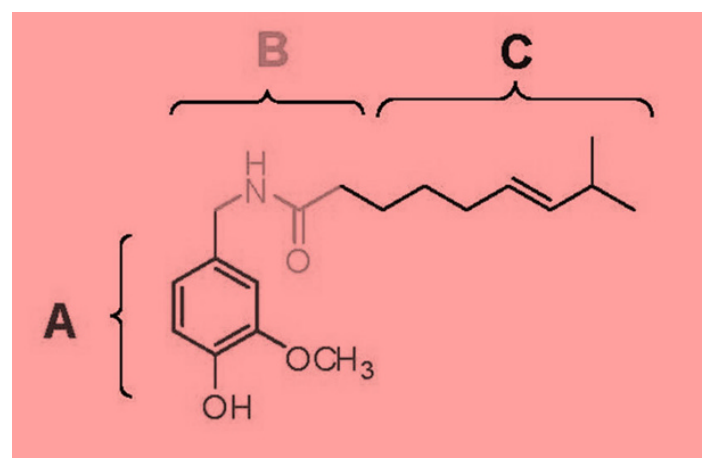

Fig. 1 Chemical structure of capsaicin

broadly studied and have entered into clinical trials (Corson and Crews 2007). In chemotherapy, plant origin drugs cause damage equally to both normal and malignant cells. By carefully designing the nanocarriers, natural product formulations can be targeted to specific cells (Sahoo and Labhasetwar 2003).

Capsaicin (8-Methyl- $N$-vanillyl-trans-6-nonenamide) is an active component of capsicum species. It is responsible for the characteristic pungent smell. It has several potential applications as anticarcinogenic [14] antioxidant (Rosa et al. 2002), suppressor of fat accumulation (Yoshioka et al. 1999), anti-inflammatory (Fraenkel et al. 2004; Fernando Spiller et al. 2008) and as antimicrobial agent (Kurita et al. 2002; Zeyrek and Oguz 2005).

Pure capsaicin is a hydrophobic, colorless, odorless, and crystalline to waxy compound. It has the chemical formula $\mathrm{C}_{18} \mathrm{H}_{27} \mathrm{NO}_{3}$, with a molar mass of $305.40 \mathrm{~g} / \mathrm{mol}$ ("ChemSpider"). The structure of the capsaicin molecule can be divided into three distinct regions, $[\mathbf{A}]$ as a vanillyl group, $[\mathbf{B}]$ an amide bond and [C]a fatty acid chain (Fig. 1) (Hopur2 2009). Due to the presence of the hydrophobic side chain, capsaicin is lipophilic and, therefore, highly soluble in fat, oil, and alcohol (Reyes-Escogido et al. 2011). It has poor bioavailability due to its hydrophobic nature like many other natural phytomedicines which is a major limitation in drug delivery. Therefore, in the present study, the capping of capsaicin on AgNPs by the direct reduction of silver nitrate in the aqueous phase, without the use of any other reducing agents was undertaken with the aim of developing a potential therapeutic drug.

\section{Materials and methods}

Synthesis of silver nanoparticles and UV-vis spectroscopy studies

Capsaicin (8-methyl- $N$-vanillyl-trans-6-nonenamide) was purchased from Sigma Chemical Co, St. Louis, MO, USA. The silver nitrate was purchased from Qualigens, Mumbai.
Glasswares were rinsed with deionised water before starting the synthesis. $20 \mathrm{ml}$ of $1 \mathrm{mM} \mathrm{AgNO}_{3}$ was rapidly stirred at $60{ }^{\circ} \mathrm{C}$ to which $100 \mu \mathrm{l}$ of $1 \mathrm{mg} / \mathrm{ml}$ concentrated solution of capsaicin was added. Silver nanoparticles gradually formed as the capsaicin reduces and binds to the surface of silver nanoparticles (SNPs). Further heating was stopped when the reaction mixture turned purple. The presence of colloidal nanoparticles in the solution was detected by passing laser beam. The bio-reduction was monitored at different time intervals of $30 \mathrm{~min}, 1,2,16$ and $24 \mathrm{~h}$ by color change and UV spectroscopy measurement.

\section{Kinetics}

UV-vis spectroscopy measurements of the silver nitrate solution, capsaicin and capsaicin-capped silver nanoparticles were carried out on a UV-vis spectrophotometer UV2450 (Shimadzu). The kinetics of bio-reduction of silver nano particles at different time intervals was recorded. The spectra from 200 to $800 \mathrm{~nm}$ were recorded for the synthesized silver nanoparticles-pellet and remaining capsaicin in the supernatant after centrifugation.

Fourier transforms infrared (FTIR) spectroscopy measurements

After complete reduction, the colloidal silver nanoparticles were centrifuged at $12,000 \mathrm{rpm}$ for $10 \mathrm{~min}$ to isolate the silver nanoparticles. The silver nanoparticles pellet obtained after centrifugation were resuspended in deionised water and again centrifuged to remove the traces of free capsaicin present in the solution prior to FTIR analysis. FTIR spectrum was taken to assess functional groups and the involvement of possible capping of capsaicin on silver nanoparticles was carried out on a Perkin-Elmer FTIR spectrum one spectrophotometer.

\section{TEM measurements}

TEM samples of the silver nanoparticles were prepared by placing a drop over carbon-coated copper grids and allowing the solvent to evaporate. TEM measurements were performed on a JEOL model 1200EX instrument operated at an accelerating voltage between 80 and $200 \mathrm{kV}$.

\section{Hemagglutination test}

This test was performed in the microtiter plate. To each well, $50 \mu \mathrm{l}$ of PBS was added. The following solutions were added in the given order. In column 1 (A-F) $50 \mu \mathrm{l}$ of PBS that serves as control without any treatment was added. To columns 2, 3 and $450 \mu$ of Anti-A, Anti-B and 

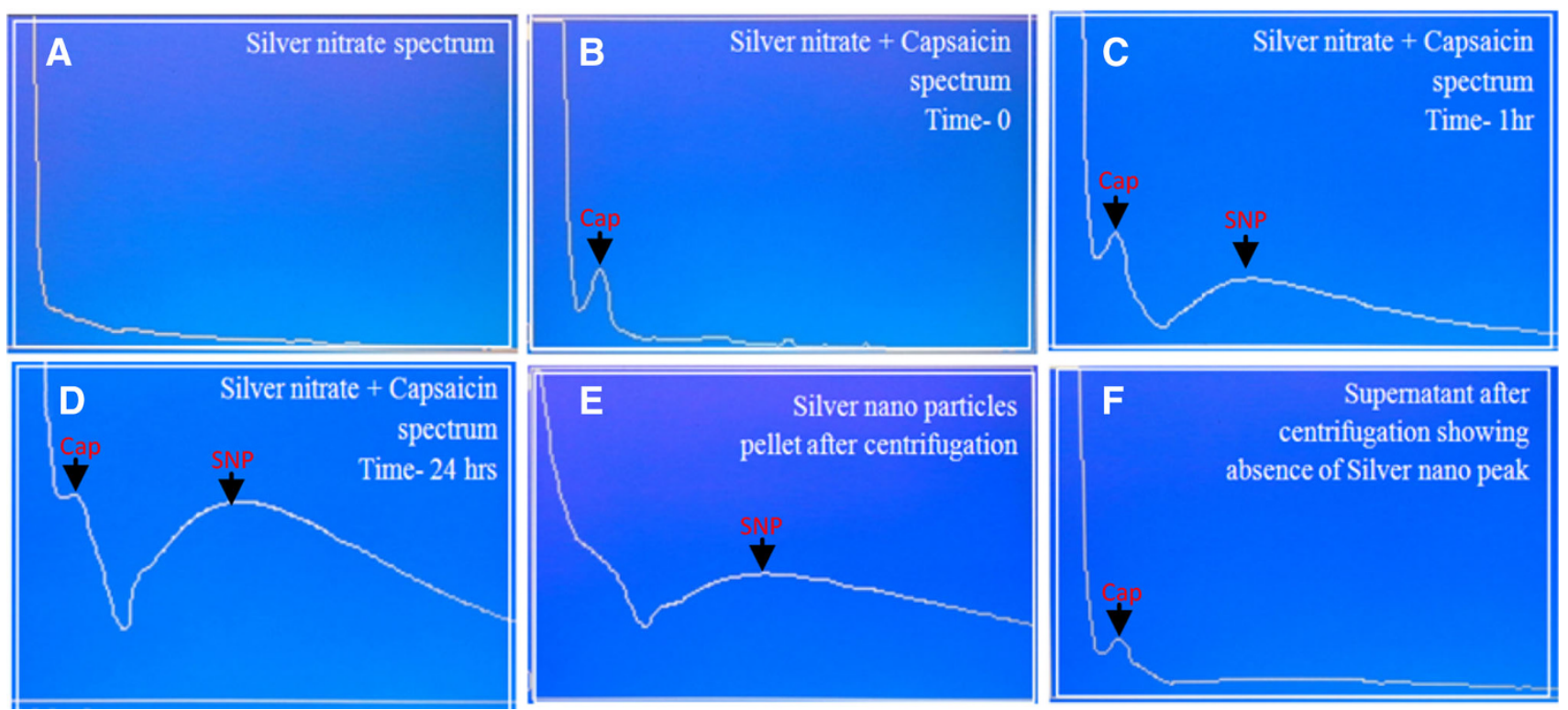

Fig. 2 Time dependent UV-vis spectra during the formation of capsaicin-capped silver nanoparticles

Anti-D were added, respectively. To the column 5, $50 \mu \mathrm{l}$ of PHA (Himedia, Mumbai) was added. In column 6 of A, C and F $50 \mu \mathrm{l}$ of capsaicin-capped silver nanoparticles was added, mixed well and $50 \mu \mathrm{l}$ transferred to the next well on its right. Repeated mixing and transfer of $50 \mu$ down the length of the plate up to column 9 was done; final $50 \mu 1$ from the last well was discarded into a bleach solution. Agglutination inhibition test was performed by mixing the anti-A, B and D with nanoparticles in columns 10-12. To each well, $50 \mu \mathrm{l}$ of $0.5 \%$ red blood cells was added. It was incubated at room temperature for 30-60 min. Negative results appeared as dots in the center of round-bottomed plates. Positive results formed a uniform reddish color across the well.

\section{Results}

Synthesis and UV-vis spectroscopy studies

Silver nitrate solution mixed with capsaicin solution showed no significant change in the UV-vis spectra at $0 \mathrm{~h}$, only the characteristic peak of capsaicin at $220 \mathrm{~nm}$ was observed in the curve Fig. $2 \mathrm{~b}$ and found to be $100 \mu \mathrm{g}$ calibrated using linear regression equation $(Y=0.00919 X-0.0084)$ of capsaicin. As the time increased decrease in the capsaicin peak at $220 \mathrm{~nm}$, with an emerging peak around $458 \mathrm{~nm}$ as well as a change in color of the solution to pale gray color indicated the reduction of silver nitrate to silver nanoparticles as shown in (Fig. 3a). Absorption spectra of silver nanoparticles formed in the reaction mixture had absorbance peak at $450 \mathrm{~nm}$, arising due to the excitation of surface plasmon vibrations in the silver nanoparticles as seen in (Fig. 2d).

\section{Kinetics}

Time taken for complete bio-reduction of silver nitrate and capping was found to be $16 \mathrm{~h}$ even after which the reduction was stationary up to $24 \mathrm{~h}$ (Fig. 2d). The UV spectrum of the nano pellet after centrifugation was devoid of capsaicin peak (Fig. 2e). But free capsaicin peak was found in the supernatant and measured about $60 \mu \mathrm{g}$ (Fig. 2f). The amount of capsaicin required to reduce $20 \mathrm{ml}$ of $1 \mathrm{mM}$ silver nitrate solution was found to be $40 \mu \mathrm{g}$ approximately.

\section{FTIR}

FTIR measurements were carried out to identify the possible biomolecules in the capsaicin-reduced nanoparticles and also the capping agents responsible for the stability of the biogenic nanoparticles solution. FTIR spectrum of capsaicin showed prominent absorption bands at 3,435, 2,932, 2,351, 1,637, 1,538,14,437, 1,026 and $812 \mathrm{~cm}^{-1}$. Capsaicin structure has an aromatic ring, a polar group and a hydrophobic moiety (Fig. 4a). FTIR was done to interpret the structural modifications that had occurred in capsaicin upon formation of capsaicin-capped SNPs. We got conspicuous peaks around 3,447, 2,922, 2,352, 1,635, and a peak around $1,025 \mathrm{~cm}^{-1}$ followed by not-so conspicuous peaklets (Fig. $4 \mathrm{~b}$ ). The bands around $3,400 \mathrm{~cm}^{-1}$ are likely due to the presence of residual water molecules or the presence of $\mathrm{OH}$ group in capsaicin. The bands around 
Fig. 3 SNPs colloidal solution (a), Tyndall effect (b) and TEM image of capsaicin-capped SNPs (c)
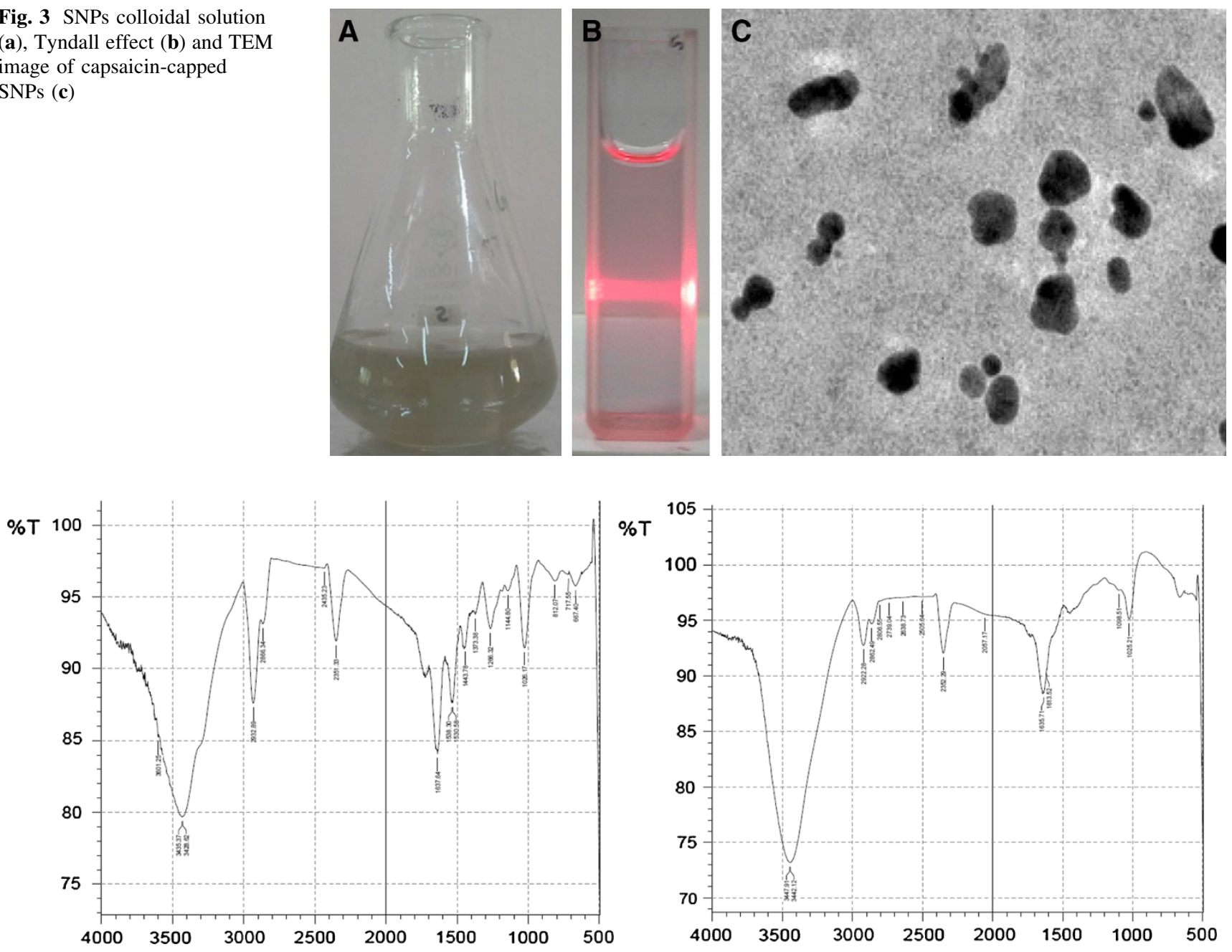

Fig. 4 FTIR transmittance spectra of capsaicin (a) and SNPs capped by capsaicin (b)

$2,750-3,000 \mathrm{~cm}^{-1}$ are likely to originate from carbonyl $\mathrm{C}=\mathrm{O}$ group present in the capsaicin. The bands around $1,600-1,750 \mathrm{~cm}^{-1}$ are likely due to the amide present in capsaicin molecule and the presence of peaklets at the lower wave numbers following it could also likely suggest the presence of aromatic $\mathrm{C}=\mathrm{C}$, which is present in capsaicin molecule, and the disappearance of the peaklets at the lower wavenumber region after the formation of SNPs suggests the possible structural change of the aromatic group. It is known that aromatic groups reduce $\mathrm{Ag}$ ions into metallic NPs, which peak around $1,646 \mathrm{~cm}^{-1}$ which is likely to be aliphatic (alkene) $\mathrm{C}=\mathrm{C}$ and whose absorption is converged with aromatic and amide absorption bands.

\section{HR-TEM measurements}

TEM measurements divulged that the resultant product after $24 \mathrm{~h}$ was composed of nearly spherical AgNPs with 20-30 nm diameter (Fig. 3c).
Hemagglutination test

One of the main aims of the current study is to increase the bioavailability of capsaicin for biological applications. The hydrophobic capsaicin is now conjugated with SNPs which is in water, hence becoming more available. The SNPs act as carrier of drug capsaicin. Before utilizing the SNPscapsaicin conjugates for clinical application, it is compulsory to confirm their biocompatibility particularly with human blood. Hence, we have performed hemagglutination (H) and $\mathrm{H}$-inhibition assay in the presence of SNPs-capsaicin conjugates. The test was conducted with $\mathrm{A}, \mathrm{B}$ and $\mathrm{O}$ blood groups. The tested blood groups showed positive results to respective test antigens. The capsaicin-capped silver nanoparticles showed negative results for the screened blood groups. But "O" positive blood group showed light agglutination when compared to " $\mathrm{A}$ " and "B" blood groups. The hemagglutination inhibition test was negative for all the blood groups. The assay result 
Fig. 5 Hemagglutination and hemagglutination inhibition test for the capsaicin-capped silver nanoparticles for $\mathrm{ABO}$ blood groups. $C$ Control, $A$ Anti-A, $B$ Anti-B, $D$ Anti-D, $P H A$ phytohemagglutinin, $N N 3$ nanoparticles dilution, $A+N P$ Anti-A and nanoparticles, $B+N P$ Anti-B and nanoparticles, and $D+N P$ Anti$\mathrm{D}$ and nanoparticles

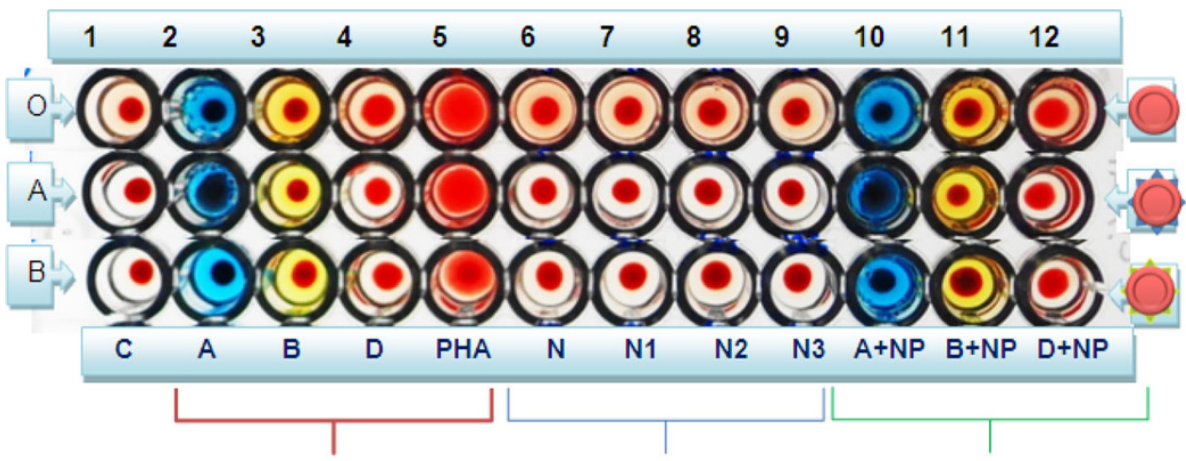

Hemagglutination test for Hemagglutination test Hemagglutination inhibition known antigen for NPs test for NPs confirms the compatibility of AgNPs-capsaicin conjugate with normal blood cells, as RBC disruption was not observed which would lead to H-agglutination (Fig. 5).

\section{Discussion}

In ayurvedic medicine, silver is used in petite amounts as a tonic, elixir or rejuvenating agent. Currently, these are also available in oral, injectable, and topical forms (Kishore Madhukar Paknikar 2008). The ash of silver, also known as Raupya Bhasma, is used to treat diseases and disorders like pain, neuralgias, inflammation, anxiety, convulsions, memory loss etc. (Nadeem et al. 1999; Hamilton et al. 1972). Moreover, nanosize of silver particle is possibly responsible for improving the absorption of silver in brain for the treatment of various pain and neurological conditions, (Khanna et al. 1997; Wang et al. 2005; Chou et al. 2001). Capsaicin has been included in topical treatments aimed at relief of different neuropathic pain syndromes such as post-herpetic neuralgia, musculoskeletal pain, diabetic neuropathy, osteoarthritis and rheumatoid arthritis (Backonja et al. 2010; Tesfaye 2009; Watanabe et al. 1987; Sawynok 2005; Derry et al. 2009). Hence our study on capsaicin-capped silver nanoparticles has a huge impact in the field of pharmacology as active drug for neurological disorders.

In vitro studies in human skin showed that capsaicin adsorption and biotransformation were found to be slow and that most capsaicin remained unaffected, while a small fraction was metabolized to vanillylamine and vanillyl acid (Suresh and Srinivasan 2010; Chanda et al. 2008; Kawada et al. 2008; Kawada and IwaiK 1985). The reason for this is varying structures of the compounds, their fair aqueous solubility, poor permeability and instability. The natural products undergo fast oxidation under basic conditions and first pass metabolism before reaching systemic circulation (Haslam 1996; Anders 2002). Many pharmaceutical companies synthesize several million compounds, but only a few of those new chemical entities become leads for preclinical development as a result of issues associated with their solubility. Nanotechnology paves way for insoluble compounds to be attached or encapsulated with highly soluble nanoparticles, offering number of drugs entry into clinical trials (McNeil 2005).

In this present study, capsaicin was found to be a potential lipophilic alkaloid which reduced the silver nitrate to silver nanoparticles. The absorption maxima of silver nanoparticles were reported in the range of 400-500 nm (Prathna et al. 2011). The plasmon resonance results of the present study were in accordance with previous reports of Vijayakumar et al. (2012) on Artemisia nilagirica, indicating occurrence of a silver band at $340-400 \mathrm{~nm}$. According to the finding of Geethalakshmi and Sarada (2012), FTIR analysis of the silver nanoparticles showed absorption peaks of reduced silver at 1,653.96 and $1,027.44 \mathrm{~cm}^{-1}$ for capsaicin-capped silver nanoparticles. In FTIR, the shifting of peaks was observed which may be attributed to the difference in capping and nature of co-ordination with the metal surface of silver (Sing et al. 2009).

Protective role of capsaicin in the gastrointestinal tract has produced controversial results and clearly indicate the need for further clinical trials to better define effective dosages (Holzer and Lippe 1988). Studies have been carried to elucidate the pharmacokinetics of curcumin as it is poorly absorbed from the gastrointestinal (GI) tract after oral administration due to its low water solubility and low stability in GI fluids. But nanoparticle formulations of curcumin exhibited increased solubility in water compared to free curcumin (Bisht et al. 2007). The orally administered capsaicin was absorbed by the tissues and concentrated in the blood. About $24.4 \%$ of administered capsaicin was circulated to liver, kidney and intestine through blood stream (Suresh and Srinivasan 2010). In our study, the capsaicin-capped silver nanoparticles tested for hemagglutination proved that they are highly biocompatible with all three types of blood groups. Hence, the 
capsaicin-capped silver nanoparticles would be a promising drug after high throughput in vivo screening.

\section{Conclusion}

Pure capsaicin lacks in bioavailability and permeability, mainly due to its poor solubility in water. To improve the bioavailability of capsaicin, it is conjugated to the surface of metal nanoparticles. In the present study, we report the binding of capsaicin to the surface of silver nanoparticles (SNPs). The nanoparticles were characterized by FTIR and TEM. The synthesized gold nanoparticles were found to be 20-30 nm in size. We found that capsaicin acts both as a reducing and capping agent, stabilizing the silver solution for 6 months. The synthesized SNPs were highly compatible with $\mathrm{ABO}$ blood groups in hemagglutination test. In vivo studies on the capsaicin-capped silver nanoparticles can be carried out to test its efficacy in the topical applications.

Acknowledgments Our grateful thank to Rev. Sr. Mercy Margret B.ICM, Mrs. Sargunam Amma and Ameer for their encouragement. Special thanks to Mr. Lourd Xavier IIT Madras Nanotechnology Unit, for his intellectual guidance and valuable suggestion. Authors thank Loyola College Management for their sustained support.

Open Access This article is distributed under the terms of the Creative Commons Attribution License which permits any use, distribution, and reproduction in any medium, provided the original author(s) and the source are credited.

\section{References}

Anders B (2002) Interaction of plant polyphenols with salivary proteins. Crit Rev Oral Biol Med 13:184-196

Backonja MM, Malan TP, Vanhove GF et al (2010) Tobias (NGX4010, a high-concentration capsaicin patch, for the treatment of postherpetic neuralgia: a randomized, double-blind, controlled study with an open-label extension. Pain Med 11:600-608

Baimark Y (2012) Preparation of surfactant-free linear and starshaped poly (l-lactide)-b-methoxy polyethylene glycol nanoparticles for drug delivery. J Appl Sci 12:263-270

Bisht S, Feldmann G, Soni S, Ravi R, Karikar C,Maitra A, Maitra A (2007) Polymeric nanoparticle-encapsulated curcumin ("nanocurcumin"): a novel strategy for human cancer therapy. J Nanobiotechnol 5. 10.1186/1477-3155-5-3

Chanda S, Bashir M, Babbar S, Koganti A, BleyK (2008) In vitro hepatic and skin metabolism of capsaicin. Drug Metab Dispos 36:670-675

Chen YY, Wang CA, Liu HY, Qiu JS, Bao XH (2005) Ag/SiO2: a novel catalyst with high activity and selectivity for hydrogenation of chloronitrobenzenes. Chem Commun 42:5298-5300

Chou CW, Chu SJ, Chiang HJ, Haung CY, Lee CJ, Sheen SR et al (2001) Temperature programmed reduction study on calcinations of nano palladium. J Phys Chem B 38:9113-9117

Corson TW, Crews CM (2007) Molecular understanding and modern application of traditional medicines: triumphs and trials. Cell 130:769-774
Derry S, Lloyd R, Moore RA, McQuay HJ (2009) Topical capsaicin for chronic neuropathic pain in adults. Cochrane Database Syst Rev CD007393

Feng QL, Wu J, Chen GQ, Cui FZ, Kim TN, Kim JO (2000) A mechanistic study of the antibacterial effect of silver ions on Escherichia coli and Staphylococcus aureus. J Biomed Mater Res 52:662-668

Fernando Spiller, Márcia K, Alves et al (2008) Anti-inflammatory effects of red pepper (Capsicum baccatum) on carrageenanand antigen-induced inflammation. J Pharm Pharm 60(4):473-478

Fraenkel L, Bogardus ST Jr, Concato J, Wittink DR (2004) Treatment options in knee osteoarthritis: the patient's perspective. Arch Intern Med 164:1299-1304

Geethalakshmi R, Sarada DVL (2012) Gold and silver nanoparticles from trianthema decandra: synthesis, characterization, and antimicrobial properties. Intern J Nanomedicine 7

Hamilton EJ, Minski MJ, Cleary JJ (1972) The concentration and distribution of some stable elements in healthy human tissues from United Kingdom. Sci Total Environ 1:341-374

Haslam E (1996) Natural polyphenols (vegetable tannins) as drugs: possible modes of action. J Nat Prod 59:205-215

Holzer P, Lippe IT (1988) Stimulation of afferent nerve endings by intragastric capsaicin protects against ethanol-induced damage of gastric mucosa. Neuroscience 27:981-987

Hopur2 (2009) Pharmacophore structure of capsaicin. http:// wikimedia.org/wiki/File:Capsaicin-Pharmacophore.jpg. Accessed 3 November 2009

Jin R, Cao YW, Kelly KL et al (2001) Photoinduced conversion of silver nanospheres to nanoprisms. Science 294:1901-1903

Kawada T, IwaiK (1985) In vivo and in vitro metabolism of dihydrocapsaicin, a pungent principle of hot pepper, in rats. Agric Biol Chem 49:441-448

Khanna AT, Silvaraman R, Vohora SB (1997) Analgesic activity of silver preparations used in Indian system of medicine. Indian $\mathbf{J}$ Pharmacol 29:393-398

Kingston DG, Newman DJ (2002) Mother nature's combinatorial libraries: their influence on the synthesis of drugs. Curr Opin Drug Discov Dev 5:304-316

Kishore Madhukar Paknikar (2008) Anti-microbial activity of biologically stabilized silver nano particles. EP1753293 A4

Kumari V, Kumar, Yadav SK (2012) Nanotechnology: a tool to enhance therapeutic values of natural plant products. Trends Med Res 7:34-42

Kurita S, Kitagawa E, Kim CH, Momose Y, Iwahashi H (2002) Studies on the antimicrobial mechanisms of capsaicin using yeast DNA microarray. Biosci Biotechnol Biochem 66:532-553

Li Z, Lee D, Sheng XX et al (2006) Two-level antibacterial coating with both releasekilling and contact-killing capabilities. Langmuir 22:9820-9823

McNeil SE (2005) Nanotechnology for the biologist. J Leukocyte Biol 78:585-594

Nadeem A, Khanna T, Vohora SB (1999) Silver preparations used in Indian system of medicine: neuropsychobehavioural effects. Indian J Pharmacol 31:214-221

Prathna TC, Raichur AM, Chandrasekaran N, Mukherjee A (2011) Biomimetic synthesis of silver nanoparticles by Citrus limon (lemon) aqueous extract and theoretical prediction of particle size. Colloids Surf B Biointerfaces 82:152-159

Priyaragini S, Sathishkumar SR, Bhaskararao KV (2013) Biosynthesis of silver nanoparticles using actinobacteria and evaluating its antimicrobial and cytotoxicity activity. Int J Pharm Pharm Sci 5(2):709-712

Reyes-Escogido ML, Gonzalez-Mondragon EG, Vazquez-Tzompantzi E (2011) Chemical and pharmacological aspects of capsaicin. Molecules 16:1253-1270 
Rosa A, Deiana M, Casu V et al (2002) Antioxidant activity of capsinoids. J Agric Food Chem 50:7396-7401

Sahoo SK, Labhasetwar V (2003) Nanotech approaches to drug delivery and imaging. Drug Discov Today 8:1112-1120

Sawynok J (2005) Topical analgesics in neuropathic pain. Curr Pharm Des 11:2995-3004

Setua P, Chakraborty A et al (2007) Synthesis, optical properties, and surface enhanced Raman scattering of silver nanoparticles in nonaqueous methanol reverse micelles. J Phys Chem C 111:3901-3907

Sing S, Patel P, Jaiswal S, Prabhune AA, Ramana CV, Prasad BLV (2009) A direct method for the preparation of glycolipid-metal nanoparticle conjugates: sophorolipids as reducing and capping agents for the synthesis of water redispersible silver nanoparticles and their antibacterial activity. New J Chem 33:646-652

Suresh D, Srinivasan K (2010) Tissue distribution and elimination of capsaicin, piperine and curcumin following oral intake in rats. Indian J Med Res 131:682-691
Tesfaye S (2009) Advances in the management of diabetic peripheral neuropathy. Curr Opin Support Palliat Care 3:136-143

Vijayakumar M, Priya K, Nancy FT, Noorlidah A, Ahmed ABA (2012) Biosynthesis, characterization and anti-bacterial effect of plant-mediated silver nanoparticles using Artemisia nilagirica. Indus Crops Prod 41:235-240

Wang X, Zhuang J, Peng Q, Li Y (2005) A general strategy for nanocrystal synthesis. Nature 431:3968

Watanabe T, Kawada T, Yamamoto M, Iwai K (1987) Capsaicin, a pungent principle of hot red pepper, evokes catecholamine secretion from the adrenal medulla of anesthetized rats. Biochem Biophys Res Commun 142:259-264

Yoshioka M, St-Pierre S et al (1999) Effects of red pepper on appetite and energy intake. Br J Nutr 82:115-123

Zeyrek FY, Oguz E (2005) In vitro activity of capsaicin against Helicobacter pylori. Ann Microbiol 55:125-127 